\title{
Special issue based on selected IEEE ONDM 2015 papers
}

\author{
Piero Castoldi $^{1}$ - Filippo Cugini ${ }^{2}$ - Tibor Cinkler ${ }^{3}$ - Pablo Pavón Mariño ${ }^{4}$
}

Published online: 1 June 2016

(C) Springer Science+Business Media New York 2016

The 19th International Conference on Optical Networks Design and Modeling-IEEE ONDM 2015 (http://ondm2015. sssup.it/)—was held in Pisa, Italy, on May 11-14, 2015.

IEEE ONDM is one of the premier venues in Europe for the optical network community to meet and exchange ideas. Along with the tradition of previous editions of this conference, ONDM 2015 addressed cutting-edge research in established areas such as optical networking, optical systems, as well as emerging topics like novel Internet architectures, new optical network architectures for the Cloud/DataCenter, wireless optical networks and photonic integrated networks.

ONDM 2015 was a 3 full-day event organized by Scuola Superiore Sant'Anna (SSSA), and Consorzio Nazionale Interuniversitario per le Telecomunicazioni (CNIT). The event includes three keynote lectures, nine invited papers, regular papers and a workshop organized by the PACE project.

ONDM 2015 was technically co-sponsored by the IFIP TC 6 (working group 6.10), by IEEE and by the IEEE Communication Society with the endorsement of the TAOS and ONTC.

The conference was also supported by Ericsson, Nextworks, Interoute and FP7 PACE project.

More than 80 high quality technical papers were submitted by researchers from 29 countries all over the world. All submitted papers were peer reviewed by the Members of the

$凶$ Filippo Cugini

filippo.cugini@cnit.it

Scuola Superiore Sant'Anna, Pisa, Italy

2 CNIT, Pisa, Italy

3 Budapest University of Technology and Economics, Budapest, Hungary

4 Technical University of Cartagena, Cartagena, Spain
Technical Program Committee and external reviewers. The authors of the accepted papers belong to 20 countries from Europe, the Americas, Africa, the Middle and Far East. Overall, 20 countries were represented at the conference by the 85 delegates that attended the event.

The accepted 35 contributions, together with 9 invited papers and 3 keynote talks were organized into 10 oral and one poster sessions.

The workshop of the FP7 PACE (Next Steps in PAth Computation Element (PCE) Architectures: From SoftwareDefined Concepts to Standards, Interoperability and Deployment) project was integrated as a session of the conference.

Three top-scored paper award were given to the three best works selected among the technical contributions presented at the oral sessions.

Authors of 13 selected papers were invited to submit an extended version of their work to a Special Issue of the Springer Photonic Network Communications (PNET) Journal.

After a thorough review process, 11 invited papers have been selected for publication.

Relevant advances are provided in the context of three main categories, focusing on network design, time-shared systems and networks, and transmission systems.

A brief summary of the accepted papers is provided next.

\section{Network design}

- In "Optimal Nonuniform Wavebanding in WDM Mesh Networks", Jingxin Wu, Suresh Subramaniam, and Hiroshi Hasegawa design an efficient solution for optimizing the number of wavebands and their sizes in optical mesh topologies. Both integer-linear program and efficient 
heuristics are proposed and evaluated through simulations.

- In "Static Protection Against Single Multicast Resource Failure in Optical WDM Networks", Dylan Davis and Vinod Vokkarane investigate multicast (i.e., point-tomultipoint) communication in optical networks. An optimal design solution is presented through Integer Linear Programming (ILP) for the static protected multicast Routing and Wavelength Assignment (RWA) problem.

- In "Effects of Signal Power Control Strategies and Wavelength Assignment Algorithms on Circuit OSNR in WDM Networks", Xue Wang, Yue Fei, Miguel Razo, Andrea Fumagalli, Miquel Garrich, Alexandre D. Andrade, Matheus S. Svolenski, and Heitor S. Carvalho focus on wavelength-division multiplexed (WDM) networks controlled by Software Defined Networking (SDN). In particular, a number of signal power control strategies is proposed to concurrently control the gain of each individual optical amplifier at each reconfigurable optical add/drop multiplexer (ROADM). Simulations and experimental results are reported to prove both feasibility and effectiveness of a coordinated use of signal power control strategies and wavelength assignment algorithms.

- In "Provisioning Virtualized Cloud Services in IP/MPLSover-EON Networks" Pan Yi and Byrav Ramamurthy focus on the provisioning of network-efficient virtualized cloud infrastructure in IP over elastic optical networks (EON). A mixed integer linear programming (MILP) formulation and a cost-optimized heuristic are proposed and evaluated through extensive simulations. Results show that sliceable transponders will reduce the total cost no matter which modulation format is adopted, and the reduction is related with the bandwidth requirement of the demands.

\section{Time-shared systems and networks}

- In "Capacity Enhanced Receivers for Low Latency Burst Optical Slot Switching Rings", Bogdan Uscumlic, Annie Gravey, Philippe Gravey, Yvan Pointurier, and Michel Morvan propose a new receiver architecture for coherent detection in slotted optical packet switching rings with elastic (rate adaptive) optical transponders. Network planning and traffic engineering simulations are provided to quantify the increase in network capacity and latency reduction that can be achieved thanks to the use of the new receivers.

- In "Novel Virtual OCS in OPS Data Center Networks", Yue-Cai Huang, Yuki Yoshida, Salah Ibrahim, Ryo Takahashi, Atsushi Hiramatsu, and Ken-ichi Kitayama propose a novel virtual optical circuit switching (VOCS) scheme for data center networks where short flows are transmitted via optical packet switching (OPS) while long flows with reliability requirements are transmitted via VOCS. Results show that, compared to OCS, VOCS has much less adverse impact on the performance of OPS, also considering a large number of virtual circuits.

- In "Performance Analysis and Transmission Strategies Comparison for Synchronous WDM Passive Star LANs", Peristera Baziana presents two synchronous transmission strategies suitable for optical WDM networks of passive star topology. Control and the data channels are designed to enable effective data packets transmission while avoiding packet collisions. The performance of both strategies are analytically studied based on Markovian models for finite population, while the performance measures are derived by closed mathematical formulas.

- In "Design and Dimensioning of Hybrid PONs", Brigitte Jaumard, Shibo Song and Rejaul Chowdhury investigate the greenfield deployment of hybrid Time Division Multiplexing (TDM) / Wavelength Division Multiplexing (WDM) passive optical networks. In particular, a threephase optimization scheme is proposed and investigated, enabling the definition of the best ONU clusterings, the placement of the upper level passive distribution node equipment, and the most appropriate passive distribution node equipment.

- In "Demonstration of $100 \mathrm{Gbps}$ optical packet switching using header processor based on 48-bit longest prefix matching", Hideaki Furukawa, Hiroaki Harai, Yasuto Kuroda, Yuji Yano, and Shoji Koyama develop a highspeed electronic header processor based on longest prefix matching (LPM) for efficient searching of optical packet destination addresses. The proposed processor has been experimentally validated on a $100 \mathrm{Gbps}$ optical packet switching system.

\section{Transmission systems}

- In "Single-Step Digital Backpropagation for Nonlinearity Mitigation", Marco Secondini, Simon Rommel, Gianluca Meloni, Francesco Fresi, Enrico Forestieri, and Luca Potì investigate nonlinearity mitigation based on the enhanced split-step Fourier method (ESSFM) for the implementation of low-complexity digital backpropagation (DBP). An effective low-complexity nonlinearity mitigation algorithm is designed and experimentally demonstrated in a $112 \mathrm{~Gb} / \mathrm{s}$ polarization-multiplexed QPSK system to achieve a transmission distance of 3200 $\mathrm{km}$ over a dispersion-unmanaged link.

- In "On the Performance of Advanced Integrated Microring Filters for Switching Applications in Next Generation Elastic Optical Networks", Claudio Porzi, Francesco Fresi, Nicola Sambo, Antonella Bogoni, and Luca Potì 
investigate the use of integrated advanced microring resonator (MRR) for switching applications in circuitswitched elastic optical networks. A practical design is proposed, enabling advantages in terms of reduced fabrication costs as well as better performance than commercial spectrum selective switch filters.

\section{IEEE ONDM 2015 General Chair}

Piero Castoldi-Scuola Superiore Sant'Anna (SSSA), Pisa, Italy, castoldi@sssup.it

\section{IEEE ONDM 2015 TPC Co-Chairs}

Filippo Cugini-CNIT, Italy, filippo.cugini@cnit.it

Tibor Cinkler-Budapest University of Technology and Economics, Hungary, cinkler@tmit.bme.hu

Pablo Pavón Mariño-Technical University of Cartagena, Spain,pablo.pavon@upct.es

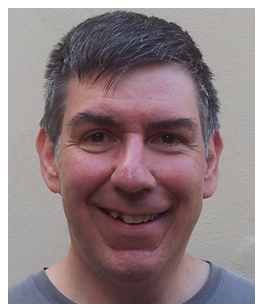

Piero Castoldi has been Associate Professor at Scuola Superiore Sant'Anna, Pisa, Italy since 2001. He is also Head of the "Networks and Services" Area at the Institute of Communication, Information and Perception Technologies (TeCIP) of Scuola Superiore Sant'Anna. Formerly he has been Coordinator of "Communication" Macro-Area at the TeCIP Institute. He has also served as Project Manager of many projects of the Inter-universitary National Consortium for Telecommunications (CNIT) and he has been Director of the National Laboratory of Photonic Networks in the period 2005-2010. He holds a Master degree cum laude in Electrical Engineering from the University of Bologna (Italy) and a Ph.D. degree in Information Technology from the University of Parma (Italy). He has also been visiting Post-doc and Researcher at the Department of Electrical Engineering at Princeton University (USA) for a total of 18 months in the period 1996-2000. He had leading roles in the following EU projects: BONE, STRONGEST, IDEALIST, OFELIA, 5GEx and FED4FIRE. His research interests cover telecommunications networks architectures, in particular control of optical networks, traffic engineering and reliability, and data center architectures for grids and clouds. He is author of more than 300 publications in international journals and conference proceedings, in addition to a single-author of a book on CDMA. He has also filed a total of 20 patents. Professor Castoldi is a Senior Member of IEEE.

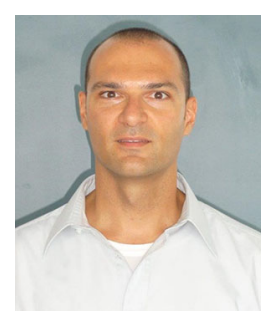

Filippo Cugini received the M.S. degree in Telecommunication Engineering from the University of Parma, Italy. Since 2001, he has been with the National Laboratory of Photonic Networks, Consorzio Nazionale Interuniversitario per le Telecomunicazioni (CNIT), Pisa, Italy. His main research interests include theoretical and experimental studies in the field of optical communications and networking. In particular, the focus is on Ethernet, GMPLS and PCE protocols and architectures, survivability and traffic engineering in IP over optical networks, multi-rate flexible optical networks, software defined networking (SDN), Segment Routing. He serves as Editorial Board Member of the Elsevier Optical Switching and Networking journal. He is co-author of twelve international patents and more than 200 international publications.

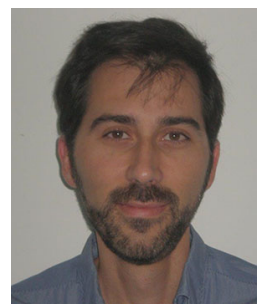

Pablo Pavón Mariño was born in Ourense (Spain). He received his Telecommunications Engineering degree from the University of Vigo (Spain) in 1999. In 2000 he joined the Technical University of Cartagena (Spain), where he received his Ph.D. degree in Telecommunications in 2004. In 2010 he received his degree in Mathematics. Since 2007, he has been working as an Associate Professor in the Department of Information Technologies and Communications of the Technical University of Cartagena. His research interests include performance evaluation and optimization of communication networks. He is the author or co-author of more than 100 papers in the field, one book, and two patents. 